\title{
The effect of contrast upon perceived speed: a general phenomenon?
}

\author{
Mark R Blakemore, Robert J Snowden \\ School of Psychology, Cardiff University, Cardiff CF1 3YG, Wales, UK; \\ e-mail: snowden@cardiff.ac.uk \\ Received 22 December 1997, in revised form 10 August 1998
}

\begin{abstract}
The perceived speed of a grating pattern has often been reported to slow as the contrast of the pattern is reduced (though there are some contradictory reports). The mechanism of this perceived slowing has not yet been established nor have the conditions under which the effect occurs (or does not occur). We have therefore examined a range of stimuli that differ upon such aspects as one versus two dimensions, periodic versus nonperiodic, and whether the stimuli occur within a static window. We have also examined a range of stimulus speeds, different types of motion, and simultaneous versus successive presentations. We have found evidence for contrast-induced changes in perceived speed in all our stimuli, and thus suggest that none of the stimulus factors listed above is critical in producing the effect. Though the pattern of results is complex and shows substantial intersubject variation, we generally found that slowly moving patterns presented simultaneously produced the greatest decrease in perceived speed with decreasing contrast. On the other hand faster speeds and successive presentation produced more veridical matches or even an increase in perceived speed with decreasing contrast.
\end{abstract}

\section{Introduction}

The relationship between the physical speed of an object and the perceived speed of that object does not always involve a simple one-to-one mapping between the two variables. A number of stimulus characteristics appear to modify the relationship (Brown 1931). These characteristics include stimulus spatial frequency (Diener et al 1976; Campbell and Maffei 1981; Smith and Edgar 1990), the nature of the background over which an object moves (Gogel and McNulty 1983; Snowden 1997), the size of the object (Brown 1931; Snowden 1997), and, of interest in the present study, the contrast of the stimulus (Thompson 1976, 1982; Campbell and Maffei 1979, 1981; Kooi et al 1992; Stone and Thompson 1992; Hawken et al 1994; Gegenfurtner and Hawken 1996; Smith and Derrington 1996; Thompson et al 1996; Thompson and Stone 1997).

Thompson $(1976,1982)$ reported that the perceived speed of a sinusoidal grating pattern was influenced by the contrast of the grating. Over a range of speeds, reducing the contrast of the grating caused a perceived slowing, though at faster speeds this relationship could be reversed. A possible explanation may lie in a model of how speed is initially encoded in the visual system. It has been suggested that speed could be calculated on the basis of a ratio between activity in a 'slow' channel and a 'fast' channel (Tolhurst et al 1973; Harris 1986). Such a model should be invariant to contrast as it takes a ratio of activity and, under most circumstances, changes in contrast would affect each channel in a similar manner. However, at very low contrasts this may break down because of 'thresholding'-type effects and, as the ratio becomes distorted, so does our perceived speed. However, the fact that the effect appears to hold across the whole range of contrasts (Stone and Thompson 1992) leads us to reject such an explanation. However, this is based on the assumption that the relative gains of both 'fast' and 'slow' channels are equal, if these are found to be nonlinear, then contrast effects may be expected over the whole range of contrasts. Further experiments have replicated the effects [though others have failed; for a discussion see Thompson (1993)] and shown that they can produce a change in the perceived direction of plaid motion (Stone et al 
1990; Ferrera and Wilson 1991; Kooi et al 1992; Stone and Thompson 1992) and plaid speed (Stone et al 1990; Gegenfurtner and Hawken 1996). Contrast has also been found to influence the perceived speed of second-order-motion stimuli (Ledgeway and Smith 1995; Gegenfurtner and Hawken 1996).

However, other researchers have suggested little influence of contrast on speed perception. McKee et al (1986) found that speed-discrimination thresholds were not affected by random variations in stimulus contrast. However, the effect of contrast on perceived speed is a speed bias whereas McKee et al examined discrimination. It is possible to have biases without affecting discrimination but the biases caused by contrast in this speed-discrimination study should have led to increases in discrimination thresholds.

Gratings are useful stimuli for studying motion perception. They are stimuli that are discrete in Fourier space, easily produced and manipulated by the experimenter, and cognate with the classical scale-based theories of visual perception (Campbell and Robson 1968). On the other hand the features that make gratings such attractive visual stimuli are also a potential limitation when studying motion perception. Three grating properties in particular may have importance in our perception of the speed: gratings are (a) temporally periodic, (b) contain conspicuous features, and (c) are contained within a 'window'.

The periodic or repetitive nature of gratings, ie the fact that a single point on the retina will be continuously stimulated over time by an identical waveform pattern, may be problematic in speed-matching tasks. This periodicity may prove to be a distinct temporal cue when matching gratings. If this is the case, gratings may be matched for temporal frequency rather than speed per se. The debate over whether we process speed independently of temporal frequency (McKee et al 1986) or whether speed is derived from temporal frequency (Smith and Edgar 1990, 1991) is still continuing. The use of nonrepetitive stimuli may avoid the confounding of temporal frequency and speed cues, or at least reduce this problem, and therefore shed some light upon this issue.

Gratings contain the conspicuous features of light and dark bars. It is therefore possible that such features could allow the observer to mentally track the positions of these features. Cavanagh (1992) reported a difference between the perceived speed of attentively tracked stimuli and the perceived speed of stimuli in which tracking was prevented - which he termed a more 'global' judgment of motion. The 'global-motion'processing system was shown to be susceptible to misperception as a function of contrast, whilst 'attentive processing' showed no such dependence on stimulus contrast. Stimuli which vary in how conspicuous their features are, and therefore how easy they are to track, may hence produce differing results. For instance in the study of McKee et al (1986), where random variations in contrast did not influence speed discrimination, it is possible that the subjects might have been able to utilise this tracking mechanism (and thus show little influence of contrast). This particular study (McKee et al 1986) utilised foveal patterns and a successive presentation of the stimuli to be judged, both of which may have aided a tracking mechanism. Studies which have involved perifoveal or peripheral presentation, and simultaneous presentations, have often revealed greater effects of stimulus contrast (Thompson et al 1995, 1996).

The fact that gratings are nearly always presented within a static window may also be of some relevance. Some interesting findings have been found when windowgrating interactions have been systematically varied. As early as 1931, Brown noted that the size of a window has an influence upon the perceived speed of a stimulus, such that a large expanse appeared to decrease the speed of the object. The 'hardness' of the window has also been found to interact with the perceived motion of the stimulus (Zhang et al 1993). Gratings within a 'sharp window' show a form of motion contrast. When the patch as a whole is kept stationary and the grating within it is moved the whole patch appears to drift in the opposite direction to the movement of 
the grating. When the window was 'soft' the grating merged with the window. In this case motion integration was observed. The patch was 'captured' and appeared to move in the direction of the motion of the grating. One can speculate that similar effects could occur when judging perceived speed rather than merely the direction of motion. Note that Stone and Thompson (1992) used 'soft'-windowed grating patches in their speed-matching study.

For these reasons a replication of the grating studies utilising some very different stimuli varying in their spatiotemporal features and other stimulus properties may allow us to address two questions. First, is the misperception of perceived speed as a function of stimulus contrast limited to, and perhaps merely an artifact of, grating stimuli? Second, if the effect does generalise to other stimuli, are some of the properties mentioned above important for the actual occurrence or the strength of the effect?

The stimuli utilised in these studies were chosen for a number of reasons, including whether or not the patterns were different from gratings on some of the criteria mentioned earlier - ie whether they were periodic, conspicuous of feature, or 'windowed'. Also, stimuli that have been commonly used in other visual studies (eg random-dot patterns) deserve to be included in the study. After all, if these stimuli are utilised in motion tasks it is important to realise any possible limitations they may impose in other studies and applied settings.

Another limitation to this area of study has been its fixation upon only one form of motion; that being movement across the frontoparallel plane. To what extent does the effect transfer to other possible motions such as rotations and expansions/contractions? Such motions can be characterised by the relative motion of points/elements within the pattern and are thought to be encoded in different areas of the brain from those involved in frontoparallel motion (Snowden 1994). Different forms of motion may be affected by contrast in very different ways. Will contrast affect the speed matching of these types of stimuli?

With these additional stimuli attention should also be paid to the results of the grating studies (eg Thompson 1982; Stone and Thompson 1992). These include the reversal of the effect at temporal frequencies of $16 \mathrm{~Hz}$ (Thompson 1982), the differences observed between simultaneous and successive presentation, and even the intersubject variability of the effect, which has also been observed in plaid studies (Stone et al 1990).

\section{Experiment 1: Speed matching of gratings over a range of speeds and contrasts}

We began this series of experiments by a simple replication of the effect of contrast on the perceived speed of grating patches (Thompson 1982; Stone and Thompson 1992). The ranges of contrasts and speeds were chosen to allow a general comparison with the stimuli used in the later experiments.

\subsection{Stimuli}

The stimuli, generated by using a Cambridge Research graphics card (type 2.3) and a Research Machines 486/66 PC, were displayed upon a Mitsubishi 20X monitor in a darkened laboratory. The stimuli were horizontal gratings drifting upward or downward presented on a background of mean luminance $20 \mathrm{~cd} \mathrm{~m}^{-2}$. The gratings were presented in circular patches with hard edges. In the 'simultaneous' condition two gratings were presented with an abrupt temporal onset and travelled in the same direction for an equal duration (randomised around a mean of $320 \mathrm{~ms} \pm 32 \mathrm{~ms}$ ). The starting phases of the gratings were randomised to reduce any colinearity cues. The gratings had a diameter of $5 \mathrm{deg}$ and were centred $3.5 \mathrm{deg}$ either side of the fixation line. Fixation was maintained by using a thin vertical line equidistant from the gratings and with a length of $2.5 \mathrm{deg}$. In the 'successive' condition the gratings were presented separately in the same spatial locations, and with the same temporal characteristics as in the simultaneous case. 
The gratings were presented in a random order separated by an interstimulus interval of $480 \mathrm{~ms}$. In both conditions the spatial frequency remained constant at $1 \mathrm{cycle} \mathrm{deg}^{-1}$ and a range of standard speeds and standard contrasts were used throughout the experiment. The contrast of the test grating was $64 \%$.

\subsection{Procedure}

Subjects sat at a viewing distance of $114 \mathrm{~cm}$ and were presented with the two gratings, both travelling either upwards or downwards. The subject judged the speed of two gratings, indicating whether the left or right pattern was travelling at the faster speed via a mouse press. The speed of the test pattern was controlled by using a QUESTroutine program (Watson and Pelli 1983) that adjusted the speed of the test grating from trial to trial via a staircase procedure depending on the responses of the subject during previous trials relating to that staircase. The initial starting point for the QUEST routine began each test speed at 3-6 dB faster than the standard speed and the QUEST could be altered in steps of $1 \mathrm{~dB}$. Within a block of trials the standard pattern was presented at four different contrast levels $(64 \%, 16 \%, 8 \%$, and $4 \%$ ), with each contrast having a separate QUEST routine. Within the block of trials each contrast level was randomly interleaved. Three standard speeds were tested $\left(0.5,4\right.$, and $\left.8 \mathrm{deg} \mathrm{s}^{-1}\right)$ with each speed being tested in a separate block of trials.

After a block of trials the probability of a 'faster' response to the test grating was plotted as a function of its physical speed for each of the standard speeds and/or contrasts. The data were fitted by probit analysis (Finney 1971) to extract the point of subjective equality. All data points plotted in figures $1-5$ are the mean of at least three such replications (and the standard errors where they are shown are also for three replications).

\subsection{Subjects}

Three subjects with normal (RJS and KW) or corrected-to-normal (MRB) vision took part in the study. Subject KW was naive to the purpose of the experiment.

\subsection{Results}

The six graphs presented in figure 1 show both the simultaneous and the successive speed matches for all three subjects. The contrast of the standard pattern is presented along the $x$-axis and the velocity match is presented along the $y$-axis. The velocity match is the adjusted test speed set by the observer relative to the actual physical speed of the standard patterns. The line at the velocity-match level of 1 therefore indicates a veridical speed match.

When comparisons between different standard contrasts are made the difference in the relative velocity-match values will be stated rather than the actual physical speed difference. For example the 4\%-contrast standard matched to a $64 \%$-contrast test pattern will be compared with the match between the test and standard at equal contrasts (both 64\%). Any decimal value stated will be the difference (faster or slower) in these velocity-match values. Such comparisons will account for the relative perceived speeds of the stimuli rather than absolute physical speeds.

In the simultaneous condition (data in the left panels of figure 1) all three subjects generally show a slowing of the perceived speed as standard contrast decreases. Thus as the contrast of the standard was reduced the subjects adjusted the test pattern to slower speeds to match the standard. The 4\%-contrast standard was perceived as being from 0.17 to 0.46 slower than the velocity-match value produced when the standard was matched at a contrast of $64 \%$. Additionally, it appears from the graphs that contrast has a greater influence on perceived speed when matching is made at slower speeds. Indeed at the fastest speed of $8 \mathrm{deg} \mathrm{s}^{-1}$ subject $\mathrm{KW}$ demonstrates almost identical velocity matching for both a high-contrast (64\%) or a low-contrast $(4 \%)$ standard.

In the successive condition (data in the right panels of figure 1) the reduction in perceived speed at lower contrasts is still apparent when the standard patterns are moving 


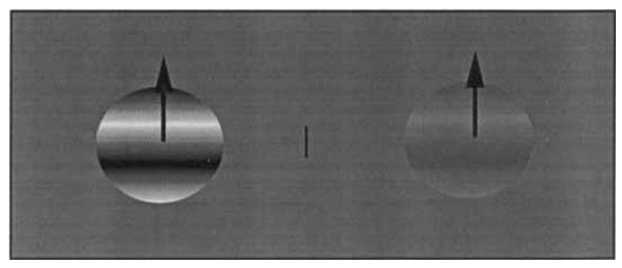

Sine-wave gratings
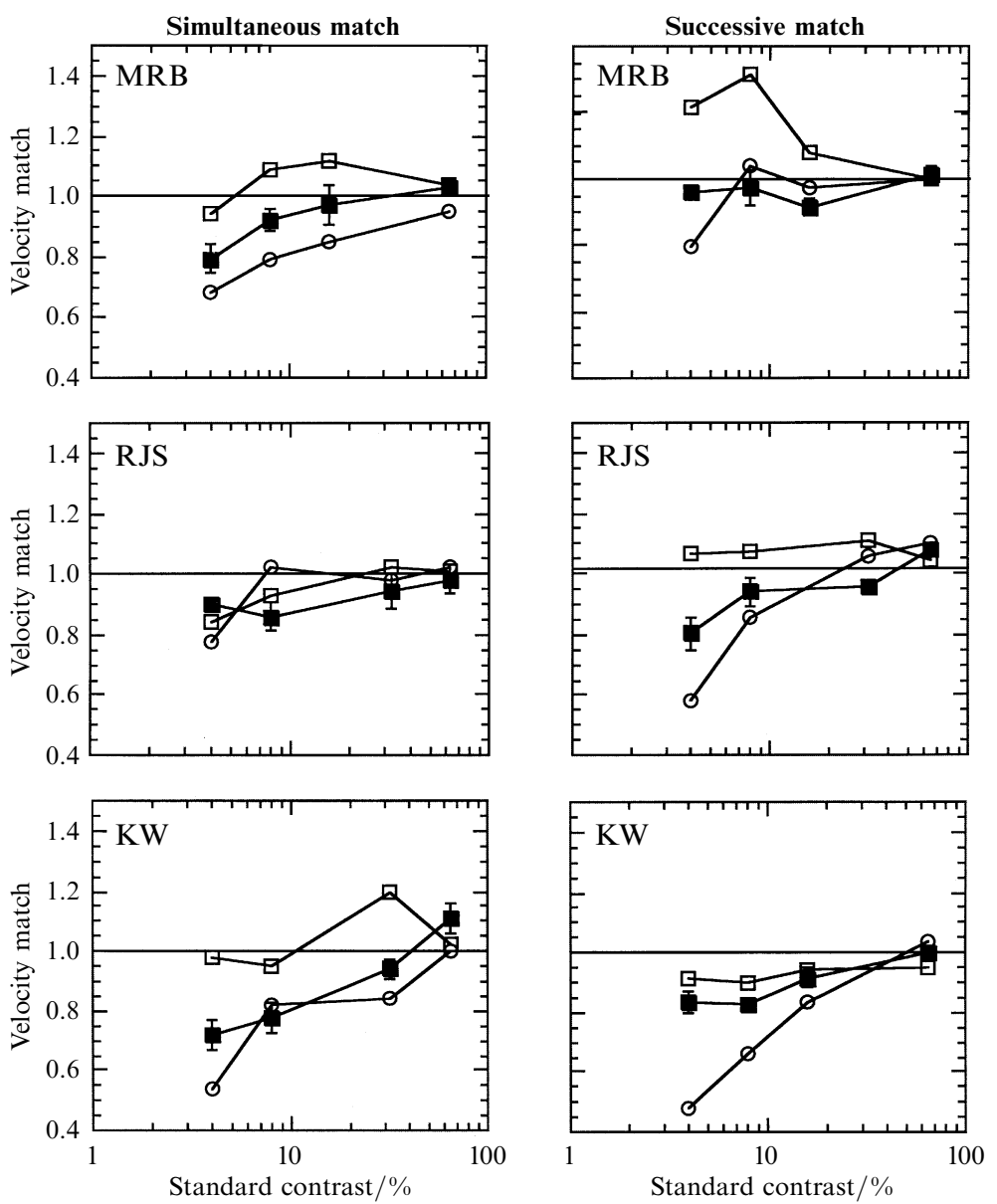

Figure 1. The speed of the test pattern (contrast of $64 \%$ ) that matched the standard contrast patterns ( $y$-axis) is plotted as a function of the standard contrast ( $x$-axis) with the speed of the test pattern as a parameter (open circles $0.5 \mathrm{deg} \mathrm{s}^{-1}$, filled squares $4 \mathrm{deg} \mathrm{s}^{-1}$, open squares $8 \mathrm{deg} \mathrm{s}^{-1}$ ). This speed is represented as a fraction of the physical speed of the standard pattern. Hence values greater than 1 indicate that the test pattern was moving faster than the standard (and for values less than 1 it was moving slower) when they were judged to have equal speed. The three panels on the left are the results of three observers under conditions where the two patterns were presented simultaneously, whilst the three on the right are for the same three observers under conditions where the two patterns were presented successively. The panel at the top gives an illustration of the stimulus but does not represent the spatial dimensions of the display. Error bars $\left( \pm 1\right.$ SEM) have been plotted only for data in the $4 \mathrm{deg} \mathrm{s}^{-1}$ condition in order to avoid unnecessary clutter. These are representative of the errors at the other speeds.

at 0.5 and $4 \mathrm{deg} \mathrm{s}^{-1}$. At the fastest speed of $8 \mathrm{deg} \mathrm{s}^{-1}$ the effect is again less pronounced (with subject RJS showing veridical matching) or even reversed, with subject MRB showing an increase in the perceived standard speed as contrast is reduced. 


\subsection{Discussion}

The results of experiment 1 were as expected, the results clearly demonstrating an effect of contrast upon the perceived speed of gratings, in agreement with the findings of Thompson (1982) and Stone and Thompson (1992). The effect was generally found to be a reduction in perceived speed of lower-contrast gratings when the gratings were presented at slower speeds. As expected, veridical matching was more likely at faster speeds and with successive presentations. In one case (subject MRB) a speeding up of the perceived speed with reduced contrast was found when the gratings drifting at $8 \mathrm{deg} \mathrm{s}^{-1}$ (and therefore at $8 \mathrm{~Hz}$ ) were presented successively. Such data were also reported in Thompson's (1982) earlier study when the gratings were presented at higher temporal frequencies.

Stone and Thompson (1992) also report that the influence of contrast upon perceived speed is more marked when the gratings are presented simultaneously rather than successively (see also Kooi et al 1992). Our data do not provide unanimous support for this finding. It appears that this manipulation had little consistent effect from subject to subject. Large intersubject differences are also striking in the data provided by Stone and Thompson (their figure 8).

\section{Experiment 2: Speed matching of Julesz patterns over a range of speeds and contrasts}

Random-dot patterns (made by randomly placing a set number of dots within an area) and Julesz patterns (made by setting each pixel to be randomly above or below the mean luminance level) have been much utilised tools for both psychophysical (McKee and Watamaniuk 1994) and physiological (Snowden et al 1992) research on motion perception. Such patterns have many properties that differ from those of a sinusoidal grating. First, they have a broad spectrum in Fourier space, containing approximately equal energy for all Fourier components (for a pattern of infinite size), whereas a grating contains only a single component (for a pattern of infinite size). Such broadband properties are common to natural scenes. If no effect of contrast is found with Julesz patterns we may assume that the effects found for gratings may be unimportant in relation to natural images. Second, the random-dot pattern is aperiodic as opposed to the periodic nature (in time and space) of a grating. Last, it has been suggested that random-dot patterns lack conspicuous features (although see McKee and Watamaniuk 1994) and thus may isolate motion mechanisms from those sensitive to position (Nakayama and Tyler 1981).

Can we make any a priori predictions as to what we might expect in this experiment? The role of spatial frequency upon perceived speed has already received some attention. For low spatial frequencies increases in spatial frequency increase the perceived speed (Diener et al 1976; Kooi et al 1992) whereas at high spatial frequencies increasing spatial frequency decreases perceived speed (Campbell and Maffei 1981; Smith and Edgar 1990). Given that random-dot patterns contain many different spatial frequencies one is faced with the problem of how the estimates arising in differing spatial-frequency bands might be combined (for discussion see Smith and Edgar 1991). Differing presentation modes might favour the subject's reliance upon differing parts of the Fourier spectrum as indeed might the subject's individual strategy (such as attention to a particular part of the spatiotemporal spectrum or deliberate covert tracking-see below). It is therefore difficult to make any precise a priori predictions about the effects of contrast upon perceived speed for these patterns.

\subsection{Stimuli}

Strips of Julesz patterns that were $3 \mathrm{deg}$ wide and $15 \mathrm{deg}$ high were presented centred $4.5 \mathrm{deg}$ either side of the fixation line. Individual pixels of the pattern were squares of side $0.05 \mathrm{deg}$. Fixation was maintained by using a thin vertical line equidistant from the dot patterns with a length of $2.5 \mathrm{deg}$. The contrast of the test pattern was 
$50 \%$ (defined as Michelson contrast). The standard pattern was presented randomly at four different contrast levels within a block of trials $(50 \%, 16 \%, 8 \%$, and $4 \%)$ and at the same speed within the same block of trials (the range of speeds being 1.5, 4, and $8 \mathrm{deg} \mathrm{s}^{-1}$ ). Presentation timings were the same as in experiment 1 .

\subsection{Procedure}

Observers judged the perceived speed of Julesz patterns moving either upward or downward. Direction of motion was randomised from trial to trial, but within a trial both patterns moved in the same direction. Measurements of perceived matching speed followed exactly the same routine as in experiment 1 .

\subsection{Subjects}

Three subjects were used: the two authors, MRB and RJS, and a naive subject KM.

\subsection{Results}

Figure 2 shows the results of both the simultaneous and the successive condition for all three subjects.

In the simultaneous conditions the lower standard speed $\left(1.5 \mathrm{deg} \mathrm{s}^{-1}\right)$ showed a perceived slowing as contrast was reduced. For subjects KM and MRB this slowing of perceived speed was only found at this slow standard speed. Matching was found to be veridical or near veridical at both 4 and $8 \mathrm{deg} \mathrm{s}^{-1}$. Additionally, both of these observers showed some decrease in the perceived speed (of around 0.1 to 0.2 ) of the $4 \%$-contrast standard pattern moving at $8 \mathrm{deg} \mathrm{s}^{-1}$, relative to the velocity match when the standard and test patterns were of equal contrast. Subject RJS on the other hand showed a stronger contrast-based misperception of speed over the range of test contrasts and standard speeds.

In successive trials the speed-matching task generally resulted in either veridical matching or a reversal of the contrast-based reduction in perceived speed over almost all the conditions. Subject MRB showed the greatest tendency to show an increase in perceived speed (relative to the 50\%-contrast standard match) as contrast was reduced with increases in perceived speed of 0.21 for $4 \%$-contrast standard gratings when matching at the faster speeds of 4 and $8 \mathrm{deg} \mathrm{s} \mathrm{s}^{-1}$.

Subject RJS on the other hand demonstrated near-veridical matching in all the conditions except the matching of the $4 \%$-contrast standard at $8 \mathrm{deg} \mathrm{s}^{-1}$. In this case the reduction in perceived speed with decreasing contrast was produced, with the $4 \%$-contrast standard perceived as 0.11 slower than the $50 \%$-contrast standard match.

\subsection{Discussion}

For the Julesz patterns the effect of contrast upon perceived speed was generally found to reduce the perceived speed at slow simultaneous speeds and reversal or veridical matching was common in faster and/or successive matches.

Observer reports suggest that the 4\%-contrast standard pattern moving at $8 \mathrm{deg} \mathrm{s}^{-1}$ may not have been clearly perceived. In the simultaneous condition subject RJS could not perceive this low-contrast pattern whilst subjects MRB and KM seem to show a rather incongruous reduction in its perceived speed, unexpected as no misperception of speed is found at the slower $4 \mathrm{deg} \mathrm{s}^{-1}$ match. Perhaps a lack of stimulus visibility may account for this perceived slowing at $4 \%$ contrast and $8 \mathrm{deg} \mathrm{s}^{-1}$.

It is clear that these patterns produced considerable intersubject variability in the effects of contrast upon perceived speed. Further there are quite marked changes between simultaneous and successive presentations. 


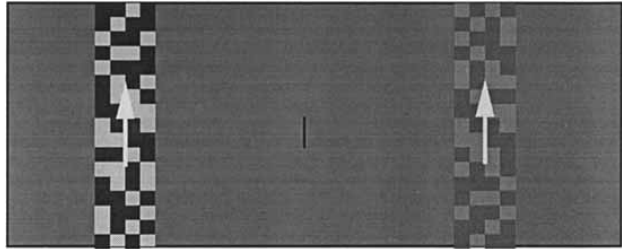

Random-dot patterns
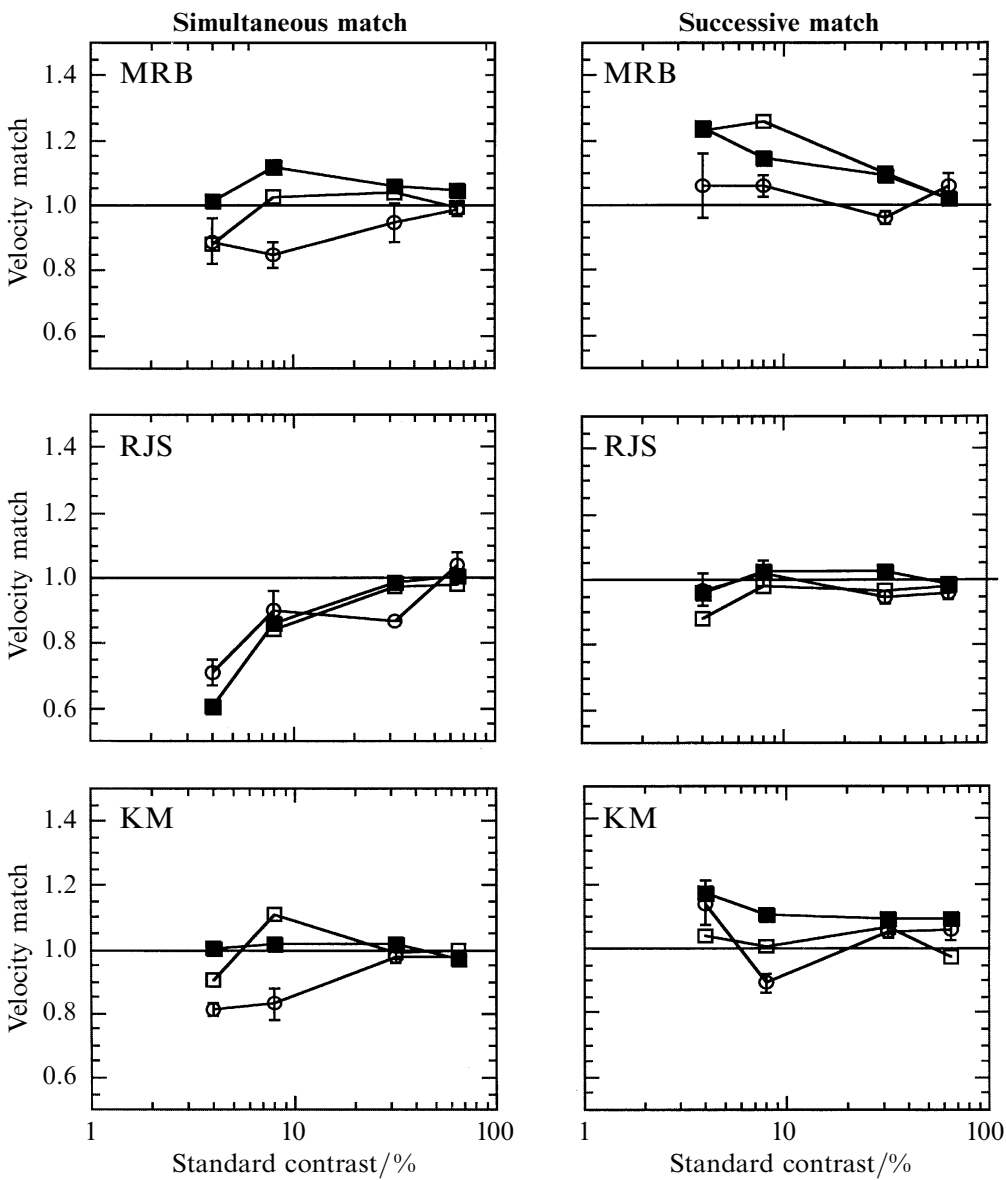

Figure 2. Speed matches for random-dot patterns. The format is as figure 1 save that the test contrast was now $50 \%$ and the standard speeds were $1.5 \mathrm{deg} \mathrm{s}^{-1}$ (open circles), $4 \mathrm{deg} \mathrm{s}^{-1}$ (filled squares), and $8 \mathrm{deg} \mathrm{s}^{-1}$ (open squares). Error bars $( \pm 1 \mathrm{SEM})$ are plotted on the $1.5 \mathrm{deg} \mathrm{s}^{-1}$ data only.

\section{Experiment 3: Speed matching of moving disc patterns over a range of speeds and contrasts} In the next experiment we were interested in whether the effect of contrast on perceived speed would remain in viewing situations that might resemble more 'natural' stimuli. We mean this in the sense that many moving objects do not move within the confines of a window and also involve a conspicuous feature changing its position (such as a car moving down a street or a bird travelling through the sky). We tested whether a simple aperiodic stimulus such as a disc would also be affected by contrast. Indeed the work of Cavanagh (1992) suggests that such a stimulus might easily engage an 'attentive tracking' mechanism that has been suggested to show little effect of contrast upon its measure of speed (Cavanagh 1992). Such aperiodic stimuli are also commonly used in other measures of motion perception such as time to collision/contact (eg Rosenbaum 
1975; Peterken et al 1991) and so, again, it is of interest to know if such stimuli are affected by or immune to these effects for practical reasons.

\subsection{Stimuli}

The discs were of diameter $2 \mathrm{deg}$ and were presented centred $2.25 \mathrm{deg}$ either side of the fixation line. A random vertical offset in their starting positions was employed of up to $2 \mathrm{deg}$ either above or below the vertical midline. The contrast of the test pattern was $50 \%$ (Michelson contrast). The standard pattern was presented at four different contrast levels $(50 \%, 25 \%, 8 \%$, and $4 \%$ ) randomly interleaved within a block of trials, and at the same speed within the same block of trials $\left(0.5,4\right.$, and $\left.8 \mathrm{deg} \mathrm{s}^{-1}\right)$.

\subsection{Procedure}

Observers judged the perceived speed of disc patterns moving either upward or downward. In both the simultaneous and the successive condition the subject judged the speed of two patterns travelling in opposite directions. This was done so that subjects could not use any change in the relative position of the two elements as a cue (eg if one element started 'behind' the other but finished 'in front' of it this would be a very obvious cue to the subject). It has previously been shown that speed-discrimination thresholds are similar when the stimuli move either in the same direction or in opposite directions [at least for randomdot patterns (Treue et al 1993)]. All other procedures were as in the earlier experiments.

\subsection{Subjects}

Three subjects took part: one of the authors (MRB) and two naive subjects (KM and IW).

\subsection{Results}

Figure 3 shows results from both the simultaneous and the successive trials for three subjects. In the simultaneous condition the speed matching was affected by the relative contrast of the disc patterns. The effect is most notable at the slower matching speeds and is nearly absent (or even reversed) for the faster speeds. In the successive condition all three observers demonstrated a reduction in the perceived speed of the $0.5 \mathrm{deg} \mathrm{s}^{-1}$ disc as contrast was reduced. Subjects IW and KM demonstrate veridical or near-veridical matching at faster speeds. However, subject MRB shows a reversal of the misperception at 4 and $8 \mathrm{deg} \mathrm{s}^{-1}$; the reduction in contrast producing an increase in the perceived disc speed.

\subsection{Discussion}

The effect of contrast upon the perceived speed of the disc patterns may be the most surprising result of the study. Again from Cavanagh's (1992) findings, one may expect a nonwindowed punctate stimulus to be less affected by contrast than the windowed periodic patterns. The former stimuli would be expected to be more readily tracked and therefore processed by Cavanagh's 'tracking' motion processors as well as the 'globalmotion' process (that is influenced by contrast). It was also a relatively strong effect, with some particularly large misperceptions at slow standard speeds and low test contrasts. Whilst these disc stimuli may engage an attentive tracking strategy it is far from certain that our subjects did this. Cavanagh (1992) was able to show that subjects could choose to use or not use this strategy according to the instructions they were given. The temporal conditions also differ considerably from Cavanagh's study (where the subjects had unlimited time to move their tracking system backwards and forwards between the two patterns and could track for as long as they pleased) and our own (brief displays). It might be that the short durations make attention tracking a less viable option.

The disc patterns are also very different from gratings in their spatiotemporal makeup; ie they are two dimensional, not windowed, aperiodic, and not discrete in their Fourier composition. Given that we have obtained strong effects of stimulus contrast upon perceived speed for both patterns this suggests that none of these factors is crucial in the emergence of this effect. 


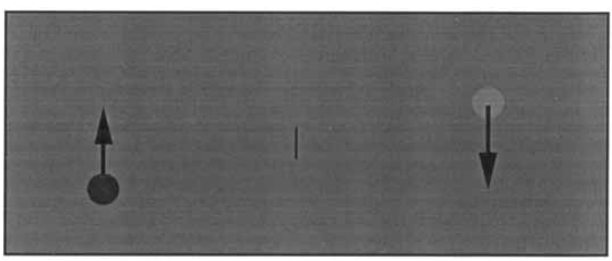

Discs
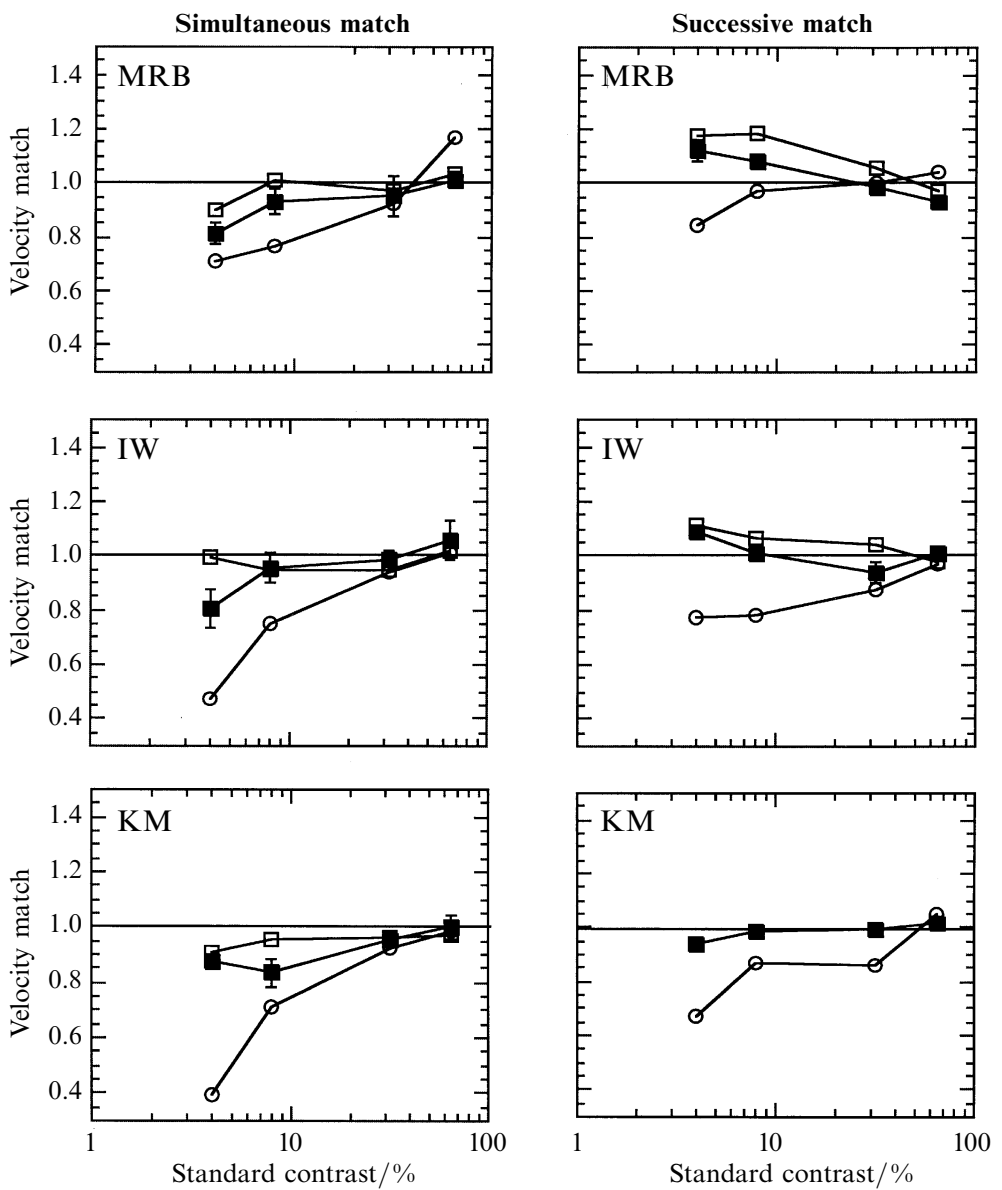

Figure 3. Speed matches for disc stimuli. The format is as figure 1 save that the test contrast was now $50 \%$.

\section{Experiment 4: Contrast and the perception of motion translation in depth}

So far the stimuli deployed in our experiments have simulated motion in the frontoparallel plane. In natural scenes motion can be more complex than this; however, there has been little study of whether the effects of contrast upon perceived speed generalise beyond this simple motion. There is some evidence that rotating stimuli do indeed show a similar effect (Campbell and Maffei 1979, 1981; Livingstone and Hubel 1988). We decided therefore to attempt to see if a simulation of a simple translation in depth [ie a disc expanding or contracting (Regan and Beverley 1978)] would also show the same effects of contrast upon perceived speed.

In recent experiments Stohr and Watamaniuk (1997) examined the perceived speed of expanding and contracting stimuli by matching their motion to that of simple linear motion. These expanding and contracting stimuli moved in a shearing fashion, ie towards 
an observer and shifting leftwards or rightwards. Expanding stimuli were matched for their rate of linear translation; that is, subjects did not use the expanding information but merely matched the linear translations of the stimulus as it moved across the frontoparallel plane. Stohr and Watamaniuk (1997) found that contracting stimuli were perceived as faster than linear discs of a constant size. This apparent difference between expanding and contracting stimuli may also be of importance when considering the effect of contrast on the perceived speed of expanding or contracting patterns. The results of Stohr and Watamaniuk suggest the possibility that contracting stimuli appear to be processed for their motion in depth and may therefore use different motion processes.

\subsection{Stimuli}

Two disc patterns were presented with their central points 3 deg either side of the fixation point. The initial sizes of the discs were separately randomised to be of diameter $2 \mathrm{deg} \pm$ up to $1 \mathrm{deg}$ (for the expanding trials) and $3.5 \mathrm{deg} \pm 1 \mathrm{deg}$ (for contracting trials). This randomisation of disc size prevented the initial size of the disc being used as a cue from which the rate of the disc expansion or contraction could be derived. The test pattern was presented at $100 \%$ contrast (dark relative to the background). Standard patterns were presented at one of four contrasts $(100 \%, 50 \%, 25 \%$, and $4 \%$ ), trials of each type being randomly interleaved within a block of trials. Two rates of radial expansion and contraction (1.5 and $3 \mathrm{deg} \mathrm{s}^{-1}$ ) were tested, each in separate blocks of trials. Here the speed refers to the linear movement at any point on the edge of the disc.

\subsection{Procedure}

This was as in the previous experiments.

\subsection{Subjects}

One author (MRB) and two naive subjects (LDT and KF) took part.

\subsection{Results}

The expanding and the contracting discs produced different patterns of results and so are presented separately (see figures 4 and 5). In the simultaneous condition a reduction in contrast is generally found to slow the perceived rate of expansion. The magnitude of the effect varies between the subjects. Subjects MRB and LDT showed a similar slowing at both test speeds, whereas subject KF showed a slowing only at the slower test speed. A somewhat similar pattern of results was obtained with the successivematching procedure. However, subject MRB appears to demonstrate veridical matching at both speeds in this case.

The results for contracting stimuli are shown in figure 5. In the simultaneous conditions decreasing contrast generally appears to reduce perceived speed and thus the results are similar to those obtained for the expanding discs. In the successive condition both subject LDT and subject KF appear to show the reversal of the effect at both speeds, ie a reduction in contrast appears to increase the perceived speed of the lower-contrast patterns. Subject MRB shows more veridical matching across the range of contrasts.

\subsection{Discussion}

For expanding patterns a reduction in contrast is generally found to produce slower perceived speeds. This effect was more robust at the slowest speed $\left(1.5 \mathrm{deg} \mathrm{s}^{-1}\right)$ and during simultaneous presentations. No reversals of the contrast effect were found to occur at these speeds.

The contracting patterns also showed an effect of contrast on perceived speed. For simultaneous patterns a slowing of perceived speed with reduced contrast was generally produced. In the successive condition a reversal of the contrast effect was generally noted at both speeds, with lower-contrast patterns perceived to contract at a greater rate than those of a higher contrast. 


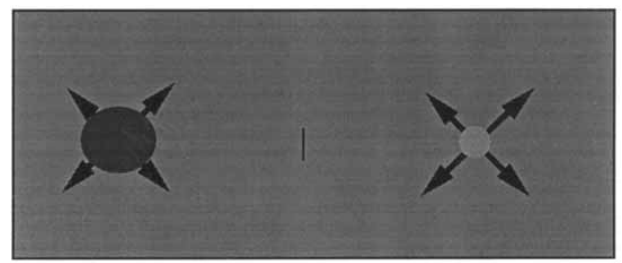

Expanding discs
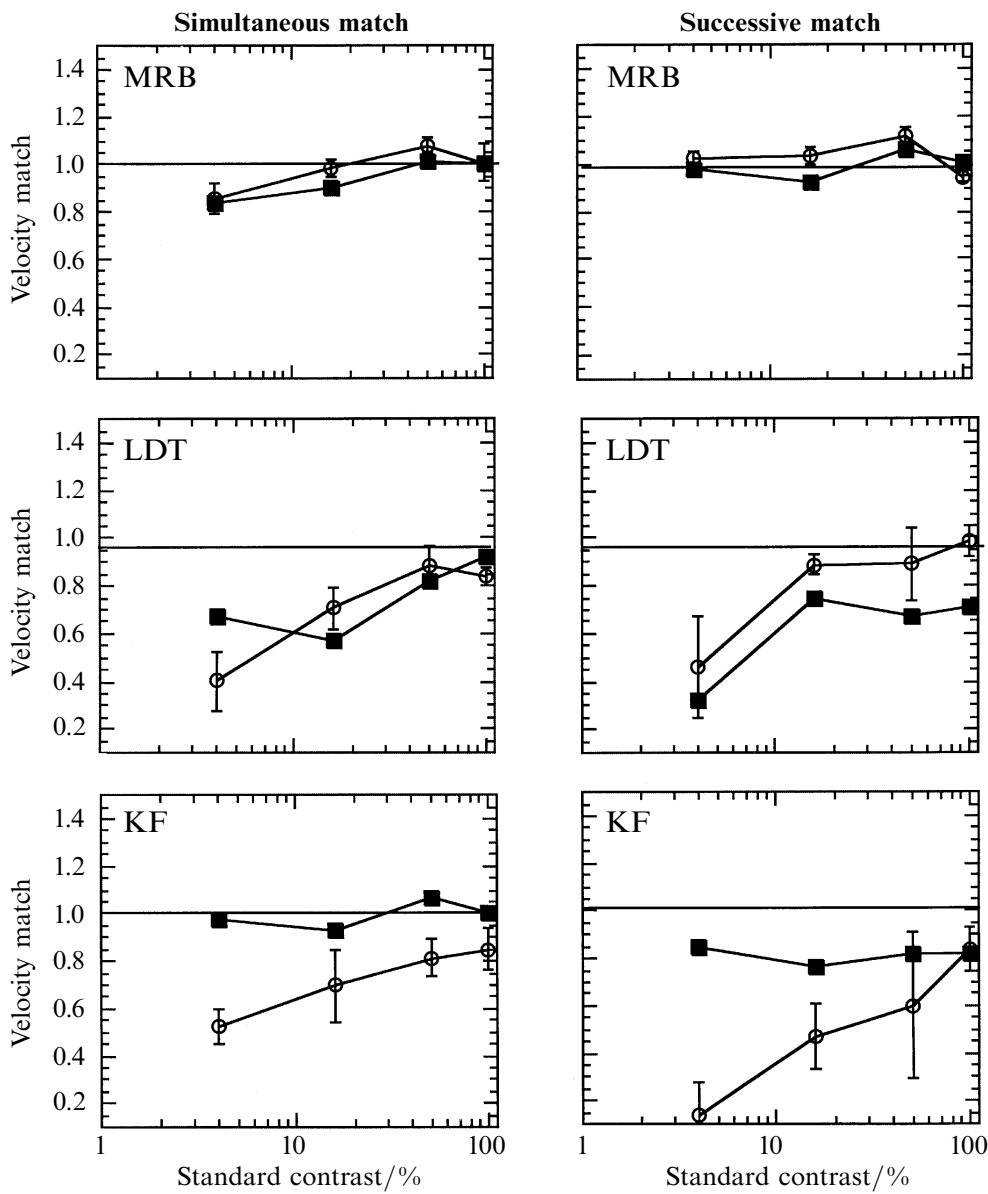

Figure 4. Speed matches for expanding discs. The format is as figure 1 save that the test contrast was now $100 \%$ and the standard speeds were $1.5 \mathrm{deg} \mathrm{s}^{-1}$ (open circles) and $3 \mathrm{deg} \mathrm{s}^{-1}$ (filled squares). Error bars ( \pm 1 SEM) are plotted on the $1.5 \mathrm{deg} \mathrm{s}^{-1}$ data only.

The results suggest that both contracting and expanding patterns interact with stimulus contrast. Results varied between the two types of motion when presentation was successive. Together with the findings of Stohr and Watamaniuk (1997) this suggests that the mechanisms for processing the two forms of motion in depth may use somewhat different algorithms. Other research also appears to support this view. Georgeson and Harris (1978) found a bias for foveofugal motion when counterphase patterns (with both expanding and contracting components) were presented in the periphery. Ball and Sekuler (1980) found that the reaction times of observers to the onset of motion are shorter for expanding patterns. Additionally, Edwards and Badcock (1993) showed that observers were more sensitive to contracting patterns than to expanding ones, despite the fact that physiological studies (Graziano et al 1994) suggest that there are more cells sensitive to expanding patterns. 

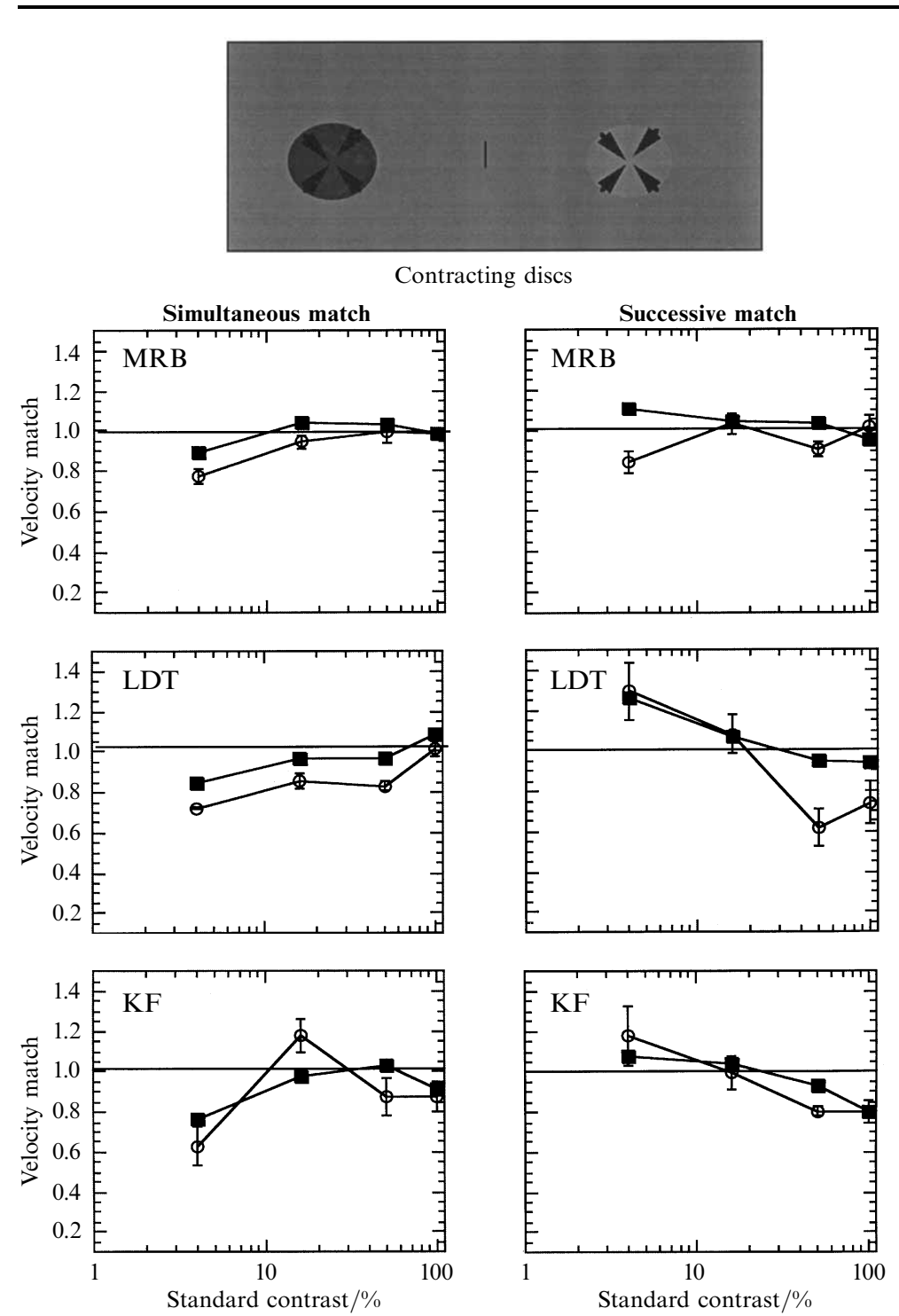

Figure 5. Speed matches for contracting disks. The format is as figure 4.

\section{General discussion}

These experiments have clearly shown that contrast can have dramatic effects upon the perception of the speed of a pattern or object and thus extend the earlier findings (Thompson 1976, 1982; Campbell and Maffei 1979, 1981; Stone and Thompson 1992; Hawken et al 1994; Thompson et al 1996). Contrast can affect match speed, with the magnitude and direction of the effect depending upon the type of stimulus and upon whether matching is successive or simultaneous. The effect also varies between subjects.

The reversal of the classic effect, an increase in the perceived speed of lower-contrast patterns, was found for at least one of the subjects in all of the experiments. This could be accounted for by the breakdown of the ratio model at threshold (Harris 1986). This model would predict that fast-moving objects should appear faster at lower contrasts. However, in the fast-speed conditions what was apparent to observers was an increase in the flicker inherent to moving stimuli. Thompson and Stone (1997) recently presented experiments that may relate this observed flicker to the reversal of the effect of contrast 
upon perceived speed. They carried out temporal-frequency-matching studies involving counterphase gratings and discovered that reducing the contrast of a pattern resulted in a perceived increase in temporal frequency though, under identical conditions, a moving grating decreased perceived speed. In experiments 1 to 4 , and indeed in the study by Thompson (1982), it could be suggested that the matching strategy used in flickering conditions was different from that used when the stimuli are slower and simultaneous. When a smooth percept of speed is not available, or indeed when flicker components cannot be ignored, observers may resort to temporal-frequency matching. This temporalfrequency matching, with its inverse relation to contrast, will produce very different matching results. Interestingly these findings also suggest that temporal-frequency matching and speed matching are different and, to some degree, separable processes; thus supporting McKee et al (1986). It is hard to reconcile the idea that speed matching is closely related to temporal frequency (Smith and Edgar 1991) when both interact with contrast in a different, indeed inverse, way.

This study does not appear to suggest any clean explanations for the effect. The wide variety of stimuli utilised in the study demonstrates the versatility of the effect, but none of the individual features of these stimuli appeared to have any consistent influence on its appearance or magnitude. Therefore whether the stimulus is one dimensional or two dimensional, periodic in nature, closely windowed, or contains conspicuous features appears of little importance.

The experiments with disc stimuli represented an attempt to test the effect of contrast upon more 'naturalistic' stimuli. We still obtained strong effects of contrast upon speed perception, suggesting that our perception of simple moving objects in 'real-life' situations may also be governed to some degree by stimulus contrast. A recent experiment (Snowden et al 1998) has also demonstrated that contrast can affect the perceived speed judgments of observers in a 'virtual' environment. In this experiment observers were instructed to drive at certain speeds in a 'virtual' vehicle. It was found that as the scene was made foggy (which reduces the contrast of the image outside the vehicle) subjects drove faster-or in other words they perceived a given speed to be slower as the scene was reduced in contrast. Similar findings were also obtained by Distler and Bülthoff (1996), who found that the perceived velocity of two simultaneously presented driving-simulation sequences was affected by the contrast and spatial frequency of the road texture.

Additionally the use of disc stimuli will allow other factors to be investigated; namely the effect of contrast upon time-to-collision measures and the effect of background reference marks upon stimuli presented at different contrasts.

We would finally like to consider what this complex pattern of results might tell us about the effect of contrast upon speed perception in general. We clearly have some situations in which one subject would reliably find a decrease in perceived speed for a particular manipulation whereas another subject would find that this same manipulation would produce no effect or perhaps even an increase. Whilst hypotheses such as contrast normalisation (Smith and Derrington 1996; Thompson et al 1996) may well have some role to play in its explanation, we suggest that 'higher-level' processes are probably of at least equal importance. For instance the extent to which the subject attempts to covertly track the stimulus may moderate any effects of contrast. This strategy is under the volition of the observer, as Cavanagh (1992) could instruct his subjects to use or not use this strategy. One might also envisage that, in displays containing many spatiotemporalfrequency components such as random-dot patterns, the subject could selectively attend to particular spatial or temporal components. As contrast has very different effects on the perceived speed of high and low temporal frequencies the extent to which the subject attends to either may be of great importance. 
Acknowledgement. Mark Blakemore was supported by an SERC research studentship (946030978).

\section{References}

Ball K, Sekuler R, 1980 "Human vision favors centrifugal motion" Perception 9 317-325

Brown J E, 1931 "The visual perception of velocity" Psychologische Forschung 14 199-232

Campbell F W, Maffei L, 1979 "Stopped visual motion" Nature (London) 278192

Campbell F W, Maffei L, 1981 "The influence of spatial frequency and contrast on the perception of moving patterns" Vision Research $21713-721$

Campbell F W, Robson J G, 1968 "Application of Fourier analysis to the visibility of gratings" Journal of Physiology (London) $197551-566$

Cavanagh P, 1992 "Attention-based motion perception" Science 2571563 - 1565

Diener H C, Wist E R, Dichgans J, Brandt T, 1976 "The spatial frequency effect on perceived velocity" Vision Research $16169-176$

Distler H, Bülthoff H H, 1996 "Velocity perception in 3-D environments" Perception 25 Supplement, 58

Ferrera V P, Wilson H R, 1991 "Perceived speed of moving two-dimensional patterns" Vision Research $31877-893$

Finney D J, 1971 Probit Analysis (Cambridge: Cambridge University Press)

Gegenfurtner K R, Hawken M J, 1996 "Perceived velocity of luminance, chromatic and non-Fourier stimuli: Influence of contrast and temporal frequency" Vision Research $361281-1290$

Georgeson M A, Harris M G, 1978 "Apparent foveofugal drift of counterphase gratings" Perception $7527-536$

Gogel W C, McNulty P, 1983 "Perceived velocity as a function of reference mark density" Scandinavian Journal of Psychology $24257-265$

Graziano M S S, Andersen R A, Snowden R J, 1994 "The tuning of MST neurons to spiral stimuli" Journal of Neuroscience $\mathbf{1 4} 54-67$

Harris M G, 1986 "The perception of moving stimuli: a model of spatiotemporal coding in human vision" Vision Research 261281 - 1287

Hawken M J, Gegenfurtner K R, Tang C, 1994 "Contrast dependence of colour and luminance motion mechanisms in human vision" Nature (London) $367268-270$

Kooi F K, De Valois K K, Grosof D H, De Valois R L, 1992 "Properties of recombination of one-dimensional motion signals into a pattern-motion signal" Perception \& Psychophysics 52 $415-424$

Ledgeway T, Smith A T, 1995 "The perceived speed of second-order motion and its dependence on stimulus contrast" Vision Research 351421 - 1434

Livingstone M S, Hubel D H, 1988 "Segregation of form, color, movement and depth: anatomy, physiology and perception" Science $\mathbf{2 4 0} 740-749$

McKee S P, Silverman G H, Nakayama K, 1986 "Precise velocity discrimination despite random variations in temporal frequency and contrast" Vision Research 26 609-619

McKee S P, Watamaniuk S N J, 1994 "The psychophysics of motion perception", in Visual Detection of Motion Eds A T Smith, R J Snowden (London: Academic Press) pp 85-114

Nakayama K, Tyler C W, 1981 "Psychophysical isolation of movement sensitivity by the removal of familiar position cues" Vision Research 21 427-433

Peterken C, Brown B, Bowman K, 1991 "Predicting the future position of a moving target" Perception $207-16$

Regan D, Beverley K I, 1978 "Looming detectors in the human visual pathway" Vision Research $18415-421$

Rosenbaum D A, 1975 "Perception and extrapolation of velocity and acceleration" Journal of Experimental Psychology: Human Perception and Performance $1395-403$

Smith A T, Edgar G K, 1990 "The influence of spatial frequency on perceived temporal frequency and speed" Vision Research $301467-1474$

Smith A T, Edgar G K, 1991 "The separability of temporal frequency and velocity" Vision Research $31321-326$

Smith D R R, Derrington A M, 1996 "What is the denominator for contrast normalization?" Vision Research $363759-3766$

Snowden R J, 1994 "Motion processing in the primate cerebral cortex", in Visual Detection of Motion Eds A T Smith, R J Snowden (London: Academic Press) pp 51-83

Snowden R J, 1997 "Perceived speed: effects of field size and background texture" Investigative Ophthalmology \& Visual Science 38(4) S1167

Snowden R J, Stimpson N, Ruddle R A, 1998 "Speed perception fogs up as visibility drops" Nature (London) 392450 
Snowden R J, Treue S, Andersen R A, 1992 "The response of neurons in area V1 and MT of the alert rhesus monkey to moving random dot patterns" Experimental Brain Research 88 389-400

Stohr R E, Watamaniuk S N J, 1997 "Size changes affect perceived speed" Investigative Ophthalmology \& Visual Science 38(4) S374

Stone L S, Thompson P, 1992 "Human speed perception is contrast dependent" Vision Research $321535-1549$

Stone L S, Watson A B, Mulligan J B, 1990 "Effect of contrast on the perceived direction of a moving plaid" Vision Research $301049-1067$

Thompson P, 1976 Velocity Aftereffects and the Perception of Movement PhD Thesis, University of Cambridge, Cambridge, UK

Thompson P, 1982 "Perceived rate of movement depends on contrast" Vision Research 22 377-380

Thompson P, 1993 "Motion psychophysics", in Visual Motion and Its Role in the Stabilization of Gaze Eds F A Miles, J Wallman (Amsterdam: Elsevier) pp 29-52

Thompson P, Stone L S, 1997 "Contrast affects flicker and speed perception differently" Vision Research $371255-1260$

Thompson P, Stone L S, Brooks K, 1995 "Speed perception in the fovea is not always independent of contrast" Perception 24 Supplement, 2

Thompson P, Stone L S, Swash S, 1996 "Speed estimates from grating patches are not contrastnormalized" Vision Research $36667-674$

Tolhurst D J, Sharpe C R, Hart G, 1973 "The analysis of the drift rate of moving sinusoidal gratings" Vision Research $132545-2555$

Treue S, Snowden R J, Andersen R A, 1993 "The effect of transiency on perceived velocity of visual patterns: a case of 'temporal capture" "Vision Research $33791-798$

Watson A B, Pelli D G, 1983 "Quest: A Bayesian adaptive psychometric method" Perception \& Psychophysics $33113-120$

Zhang J, Yeh S, De Valois K K, 1993 "Motion contrast and motion integration" Vision Research $332721-2732$ 